\title{
Investigating Effects of Post-Selection Feedback for Acquiring Ultra-Small Targets on Touchscreen
}

\author{
Chun Yu ${ }^{1}$ Hongyi Wen $^{1} \quad$ Wei Xiong $^{1} \quad{\text { Xiaojun } \text { Bi }^{2} \quad \text { Yuanchun Shi }}^{1}$ \\ Key Laboratory of Pervasive Computing, Ministry of Education \\ Tsinghua National Laboratory for Information Science and Technology \\ ${ }^{1}$ Department of Computer Science and Technology, Tsinghua University, Beijing, 100084, China \\ ${ }^{2}$ Google Inc. Mountain View, CA, USA \\ \{chunyu@,wenhy12@mails, shiyc@\}tsinghua.edu.cn, \{xio399wei,xjunbi\}@gmail.com
}

\begin{abstract}
In this paper, we investigate the effects of post-selection feedback for acquiring ultra-small (2-4mm) targets on touchscreens. Post-selection feedback shows the contact point on touchscreen after a user lifts his/her fingers to increase users' awareness of touching. Three experiments are conducted progressively using a single crosshair target, two reciprocally acquired targets and 2D random targets. Results show that in average post-selection feedback can reduce touch error rates by $78.4 \%$, with a compromise of target acquisition time no more than $10 \%$. In addition, we investigate participants' adjustment behavior based on correlation between successive trials. We conclude that the benefit of post-selection feedback is the outcome of both improved understanding about finger/point mapping and the dynamic adjustment of finger movement enabled by the visualization of the touch point.
\end{abstract}

\section{Author Keywords}

Touch behavior; Ultra-small targets; Post-selection feedback.

\section{INTRODUCTION}

Past research has investigated the impact of "fat finger" problem for acquiring small targets on touchscreens. A " $\mathrm{fat}$ finger" not only occludes targets but also imposes great touch ambiguity due to the round shape and softness of the fingertip [1]. The "fat finger" problem, associated with other factors such as target size [5], touch posture [3], visual angle [22] and etc., together complicate users' mental model of the relationship between finger positioning and anticipated touch contact point (for short, finger/point mapping model). Such complexity even makes it difficult for users to learn by practicing. Therefore, acquiring small targets with touch is imprecise. Empirical studies showed that touch accuracy (hit ratio) dropped below 40\% when target size was $2.4 \mathrm{~mm}$ [5].

Permission to make digital or hard copies of all or part of this work for personal or classroom use is granted without fee provided that copies are not made or distributed for profit or commercial advantage and that copies bear this notice and the full citation on the first page. Copyrights for components of this work owned by others than ACM must be honored. Abstracting with credit is permitted. To copy otherwise, or republish, to post on servers or to redistribute to lists, requires prior specific permission and/or a fee. Request permissions from Permissions@acm.org.

CHI'16, May 07-12, 2016, San Jose, CA, USA

(C) 2016 ACM. ISBN 978-1-4503-3362-7/16/05 ..\$15.00

DOI: http://dx.doi.org/10.1145/2858036.2858593
Various techniques have been proposed to improve touch accuracy, e.g. by decoupling motor and visual space $[1,19$, 26]), or enlarging targets to comfortable size via multi-step interactions [21]. Although effective, these techniques sacrifice the simplicity and intuitiveness of direct touch, and largely reduce efficiency. For example, small-screen software keyboards based on such techniques [15, 16] achieved only $25 \%$ of the input speed of direct input techniques $[3,25]$.

Post-selection feedback [28] serves as an orthogonal approach that augments human's basic touch ability without sacrificing the directness of touch input. It provides feedback informing the result of the touch operation, with which users can calibrate their finger/point mapping model. As shown in Fig. 1, post-selection feedback renders $\mathrm{X} / \mathrm{Y}$ coordination position of the actual contact point after a user lift up his/her finger. Typically, if a touch misses the target, the user will adjust his/her touch posture to perform better in next selection. Thus, the purpose of post-selection feedback is not to facilitate the current selection but to improve future selections. This technique can be easily implemented as system-level feature without modifying individual application.
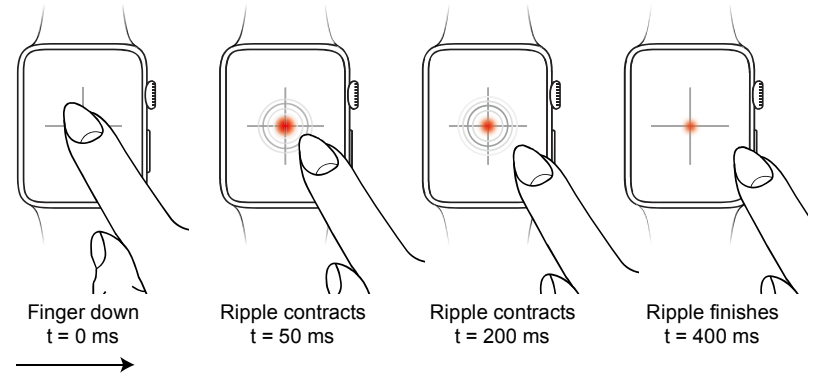

Figure 1. An example of post-selection feedback on smart watches

In this paper, we investigate the effect of post-selection feedback for acquiring ultra-small targets sized from 2 to 4 $\mathrm{mm}$. We choose this range of target size for two reasons. First, the small screen size of wearable devices (e.g. smart watches and wristbands) definitely limits the size of targets. For example, key size of a presumable QWERTY keyboard on a $42 \mathrm{~mm}$ Apple's Watch will be only around $2.2 \mathrm{~mm}$. Meanwhile, on touchscreens that have an even larger size 
(e.g. smart phones), there also exist ultra-small targets (e.g. the height of some URLs in mobile web browser is only $2 \mathrm{~mm}$ ). Therefore, extending the limit of target size that users can touch with reasonable accuracy should be of great practical interest. Second, the "fat finger" problem should be more severe for acquiring smaller targets. Hence, we assume post-selection feedback should have a more significant effect for ultra-small targets, which has not been investigated by previous research. Finally, we hope to gain deeper insights about human's touch ability by researching such small targets $[13,14]$.

To provide a comprehensive understanding, we progressively carry out three lab experiments. The first experiment requires participants to target a crosshair. We use this simple task to research users' basic touch ability. The second experiment requires participants to acquire two horizontally aligned targets back and forth. We examine how movement amplitude and target size affect the performance of post-selection feedback. The third uses a more realistic task; it requires participants to touch 2D targets randomly positioned on a smart watch under both sitting and standing conditions.

Based on the experimental data, we conduct two types of analysis. The first, as prior research did [5, 8], focuses on speed and accuracy of target selection as well as the spatial distribution of touch endpoints. Results show that with an acceptable compromise of acquisition time less than $10 \%$, post-selection feedback offers a substantial improvement on touch accuracy. Specifically, the average touch accuracy is $90.4 \%$ with post-selection feedback, and $53.8 \%$ for the nofeedback condition. In the meantime, post-selection feedback can almost remove the systematic offset and significantly reduce variance of touch endpoints by $32 \%$ at least.

The second analysis provides insights into users' touch behavior by examining the statistical relationship between immediately successive touch endpoints. The aim is to conceptualize the role of post-selection feedback in affecting users' touch behavior, or users' finger/point mapping model. Interesting results are found: with postselection feedback, no correlation $\left(\mathrm{R}^{2}<0.01\right)$ is observed between successive touches; however, in no-feedback condition, a significant correlation $\left(\mathrm{R}^{2}\right.$ as high as 0.61$)$ is found. To complete the understanding, we further develop a feedback adjustment model, based on which we examine how touch adjustment is performed in different feedback conditions. Our conclusion is that without feedback, users' finger/point mapping model is ambiguous: the systematic offset is unstable and dynamically drifted. In contrast, postselection feedback can effectively eliminate such ambiguity. As a result, touch accuracy can be improved.

\section{RELATED WORK}

\section{Human ability for acquiring small targets}

Selecting small targets on touch screen is difficult and inaccurate due to the "fat finger" problem. According to empirical studies, error rates of finger touch input were 5\% for $10.5 \sim 11.5 \mathrm{~mm}$ targets [26, 27], 12\% 27\% for $5 \mathrm{~mm}$ targets and $66 \%$ for $2.4 \mathrm{~mm}$ targets $[5,8]$.

It is widely assumed that the distribution of touch endpoints regarding a specific target is normal [5], which can be described by the mean value and deviation of the distribution. The mean coordination of endpoints is regarded as the systematic bias (offset) of users' finger/point mapping model. Researchers found that the systematic offset was linked to a number of factors. Azenkot and Zhai [3] reported different hand postures presented distinct endpoint distribution patterns for text entry on mobile phones. Holz and Baudisch [13] used crosshair targets to examine users' basic mental model of finger/point mapping. Results showed the systematic offset was highly dependent on angles of pitch and yaw, and individual users. In their later work [14], they developed a mental model stating that users aligned targets with visual features at the horizontal center of their finger outline and the vertical center of fingernail. Henze et al. [10] showed that the systematic offsets were location-dependent on touch screen.

\section{Techniques for selecting small targets}

There are various on-screen techniques improving accuracy of touching small targets. The Offset Cursor [19] positions a software pointer slightly above the finger's contact point to indicate selection position. It uses Take-off strategy to allow users to drag the pointer into the target. Shift [26] creates a callout showing a copy of the occluded screen area and places it in a non-occluded location. TapTap [21] uses two taps to indicate a selection on touch screen devices. The first tap allows an area of interest on the screen to be magnified, and the second tap select the desired target at comfortable size. ZoomBoard [16] enables users to interactively zoom the keyboard to comfortable size. CrossLever [1] and LinearDragger [2] allow users to define a region for interaction, and then perform indirect controlling by touching surrounding areas.

Off-screen techniques resolve finger occlusion by enabling users to input above, surrounding, and even behind the touchscreen surface. To enable above surface interaction, Harrison and Hudson [9] used magnetic sensors to sense finger movement. High-accurate target selection can be achieved by controlling the control/display ratio. Hover Cursor [17] takes advantage of capacity sensor of touchscreen to sense the hovering finger. A cursor can be displayed without the user touching the screen. SideSight [7] uses proximity sensors to detect users' finger input on the side of devices. Baudisch and Chu [4] explored the back-of-device interaction on small devices by attaching a capacitive touchpad to the backside of the small device. 
Users interact on the backside to avoid finger occlusion. A virtual pointer representing finger position is rendered on the front screen with 1:1 mapping. User studies showed that error rates could be reduced to $5 \%$ for $1.4 \mathrm{~mm}$ targets, and $11 \%$ for $0.6 \mathrm{~mm}$ targets. Su et al. [23] augmented fingernail with mounted display to enable always-available visual feedback. When interacting with another touch device, the fingernail screen displays image of device interface beneath the fingertip as well as software pointer to indicate the touch location.

\section{Multimodal Feedback}

Multimodal feedbacks (e.g. audio [11] and tactile [20]) can also be leveraged to improve touch accuracy. Hoggan et al. [12] defined a set of tactile signals to represent software keyboard events (e.g. fingertip-over, finger-click and fingertip-slip). Experimental results showed tactile feedback could significantly improve text entry accuracy in both static and mobile environments. Brewster et al. [6] designed different tactile signals to represent correct selection, a finger slip, and double tap errors. Experimental results showed that both accuracy and input speed of text entry could be improved, and that users were more satisfied with the presence of tactile feedbacks.

\section{Post-selection Feedback}

To our knowledge, two pieces of previous research have involved post-selection feedbacks. Wigdor et al. [28] proposed Ripples as a general visualization technique to provide feedbacks for a variety of touch interactions such as single-finger and multi-finger touching, sliding and dragging. Their investigation shows that ripple-like visual effect is effective, which could substantially reduce selection errors for $20 \mathrm{~mm}$ targets. Building on Wigdor et al.'s work, we advance the understanding of the effects of post-selection feedback. The differences from Wigdor et al.'s work are 1) we focus on ultra-small target acquisition (e.g., $2 \mathrm{~mm}$ to $4 \mathrm{~mm}$ ) on phone/watch-size devices, while Wigdor et al. investigated Ripples as a general visualization technique for tabletop interaction where the targets are usually much bigger (e.g., $20 \mathrm{~mm}$ in their study). 2) the three experiments allowed us to carry out in-depth analysis of users' touch behaviors. Based on the analysis, we, for the first time, proposed the feedback adjustment model to describe the pattern of touch point distribution and users' touch behaviors when post-selection feedback is provided.

Henze et al. [10] published a typing game to record how users touch on the standard Android keyboard. Millions of touch data were collected, from thousands of installations with screen size from 3.2 to 4.3 inches. The authors investigated three augmenting techniques (i.e. shifting touch positions, shifting key labels, and showing touch positions using dots), with the third, dot feedback, providing post-selection feedback showing the actual touch contact point. Results showed that the dot feedback could significantly reduce error rate (between $3.8 \%$ and $18.3 \%$ ) no matter used alone or combined with other techniques.
However, it also reduced input speed to $5.2 \%$, and decreased the overall performance (between $0.8 \%$ and $3.5 \%)$.

We recognize two major limitations of previous research regarding post-selection feedback. First, no research has been conducted to examine its effect for acquiring ultrasmall target sized from $2 \mathrm{~mm}$ to $4 \mathrm{~mm}$. Although it is no surprising that the effect will remain or even increase for such small targets, it is still necessary to quantify the benefits and give concrete numbers. Second but more important, previous research did not provide detailed analysis about the spatial distribution of endpoints with and without post-selection feedback. Therefore, it was difficult to draw further insights about how post-selection feedback affected users' touch behavior as [3,13, 14] did. These two limitations together motivate the current research.

\section{EXPERIMENT ONE: CROSSHAIR TARGET}

The goal of this experiment was to confirm that participants could take advantage of post-selection feedback to improve touch input accuracy. As the first step, we used a single crosshair target $[13,14]$ to make the task as simple as possible. We hypothesized post-selection feedback could improve touch precision but slow down input speed.

\section{Participants and apparatus}

We recruited 16 participants (9 male, 7 females, all right handed) between the ages of 20 and 29 from Tsinghua University. All of them used a touchscreen phone on a daily basis for more than two years. They completed the experiment with their dominant hands.

The experiment was conducted on Samsung Galaxy S3, with a screen size of $60 \times 106 \mathrm{~mm}$ and a resolution of $1280 \times 720$ pixels $(12.047$ pixels $/ \mathrm{mm})$. The experimental software reported touch data including touch-down, lift-up and finger-movement events. According to our own test, the coordinate of touch endpoint was reported as the centroid of finger contact area; there was no system-level offset manipulation.

A crosshair target $(r=30$ pixels $)$ was displayed at the center of the screen. For post-selection feedback, once a touch down event was detected, the software triggered a Point effect at the touch endpoint coordination. The initial size of Point circle was $1 \mathrm{~mm}$, and gradually contracted to a point in $400 \mathrm{~ms}$. We chose the ripple-like post-selection feedback in the study because it is now a widely used technique on touchscreen devices (e.g., Android, and Windows Phone). We used an audio feedback to notify participants that a touch event had been received by the system, regardless of selection results (successes or errors). Participants were required to lift up their finger high enough after each trial to fully see the crosshair and post-selection feedback if presented. Finally, to remove unintentional touch events (such as palm contact or fingertip tremble), touch endpoints that were $4 \mathrm{~mm}$ away from the target center or less than 
$300 \mathrm{~ms}$ from the last touch event were not responded. The same setting was used in the following two experiments.

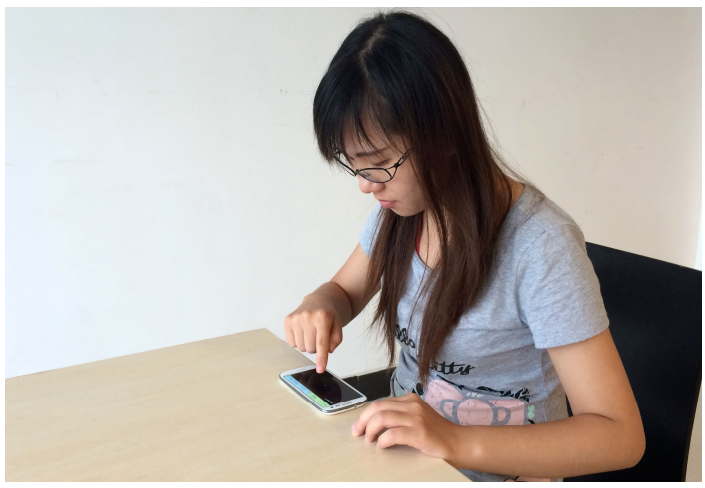

Figure 2. The experimental setting

As shown in Fig. 2, the touch screen device was positioned at a fixed location on the table. To remove uncertainty of parallax effect [22], we asked participants to keep their head above the center of the screen, and their line of sight perpendicular to the screen during the entire experiment. Participants completed the experimental task using index fingers, with their arms lifted up to approximate a more realistic scenario of using ultra-small devices (e.g. smart watches).

\section{Design and procedure}

A within-subject experiment design was used. The single independent factor was Feedback (PS feedback and no-PS). For each Feedback condition, participants were required to repeatedly acquire the crosshair for 52 times, with the first four taken as practice. The instruction given to participants was to "acquire the crosshair as accurately as possible". We counterbalanced the order of Feedback condition among participants. This design resulted in $48 \times 2 \times 16=1536$ total trials for data analysis.

Before the experiment, participants were required to familiarize themselves with the experimental tasks. They tried with both PS feedback and no-PS conditions to figure out the effective strategy to acquire the crosshair target. This warm-up session spanned 5-10 minutes. At the end of each condition, participants took a break for three minutes. After the experimental tasks, we asked participants to reflect on their strategy for taking advantage of postselection feedback.

\section{Results}

For simplicity, we denote $\mathrm{x}$-axis offset (from the target center) as dx, $y$-axis offset as dy for short in the remainder of this paper.

\section{Target acquisition time}

Paired t-test showed a significant effect of Feedback on target acquisition time MT $(\mathrm{t}(15)=2.12, \mathrm{P}<0.05)$. Acquiring targets with PS feedback was 9.7\% slower than no-PS (PS feedback: Mean $=620 \mathrm{~ms}, \mathrm{SD}=139 \mathrm{~ms}$; no-PS: Mean $=$ $565 \mathrm{~ms}, \mathrm{SD}=111 \mathrm{~ms} ;)$. This result was also consistent with participants' report that it required more mental effort to calibrate touch point when utilizing post-selection feedback.

\section{Endpoint distribution}

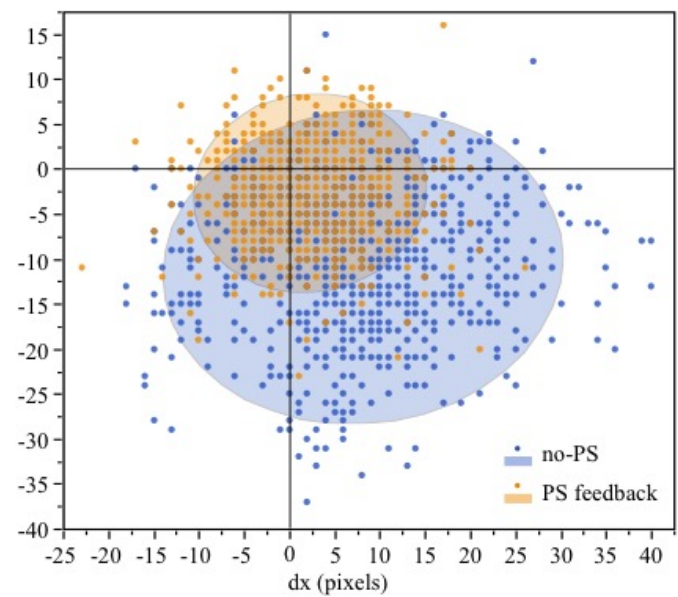

Figure 3. Endpoint distribution for PS feedback and no-PS conditions in Experiment 1. The circle covers $90 \%$ endpoints for each condition.

Fig. 3 illustrates the endpoint distribution for both PS feedback and no-PS conditions. As shown, the systematic offset lies beneath and to the right of the target center, which is in agreement with previous observations for righthanded users $[3,10]$.

Acquiring targets with PS feedback was significantly much more accurate than with no-PS. Paired t-test showed a significant effect of Feedback on endpoint offset $(\mathrm{dx}: \mathrm{t}(15)=$ $2.95, \mathrm{P}<0.01 ; \mathrm{dy}: \mathrm{t}(15)=-6.78, \mathrm{P}<0.001)$. The systematic offsets were $\mathrm{x}=8.0$ and $\mathrm{y}=-10.8$ pixels for no-PS, and $\mathrm{x}=2.2$ and $y=-2.6$ pixels for PS feedback. This means postselection feedback reduced systematic offsets by $74 \%$ in average.

The overall standard deviation of endpoint offset of PS feedback $(\mathrm{x}=6.0$ pixels, $\mathrm{SD}=2.44$ pixels; $\mathrm{y}=5.2$ pixels, $\mathrm{SD}=2.10)$ was $38 \%$ smaller than that of no-PS $(\mathrm{x}=10.3$ pixels, $\mathrm{SD}=9.02$ pixels; $\mathrm{y}=8.15$ pixels, $\mathrm{SD}=5.16$ pixels). Paired t-test showed Feedback had no significant effect $(\mathrm{t}(15)=-0.11, \mathrm{P}=0.46)$ on $\mathrm{x}$-axis deviation, but had one $(\mathrm{t}(15)=-4.17, \mathrm{P}<0.001)$ on $\mathrm{y}$-axis deviation.

Comparing PS feedback and no-PS conditions, we confirmed that individual participants had distinct systematic endpoint offsets [13], and post-selection feedback could significantly reduce the difference by making the mean endpoint of individual participants much closer to target center.

\section{Effect of presentation order}

To examine if there was any asymmetric skill transfer effect, we tested the effect of presentation order on movement time and systematic offsets for both PS feedback and no-PS conditions. Results showed no significant effects. As such, the hypothesis of asymmetrical skill transfer effect was not supported. According to our own 
observation, touch skill learned with post-selection feedback was rather ephemeral, and had to be reinforced continuously. This again reflected the complexity of finger/point model, which was not easily learnt. However, whether the skill could be transferred to no feedback condition after a long time of practice is still a question that deserves pursuit in the future.

\section{User feedback}

All participants reported that post-selection feedback could improve their understanding of the finger/point mapping. Some were surprised to see their systematic offset was that large in no-PS condition. Besides, all participants reported a similar strategy of utilizing post-selection feedback: If an offset occurred, they would perform an adjustment toward opposite direction to compensate the offset.

\section{EXPERIMENT TWO: RECIPROCAL TASK}

The goal was to examine how target size and movement distance would affect the performance of post-selection feedback.

\section{Apparatus and Participants}

This experiment involved the same participants as Exp. 1. The Samsung Galaxy 3 smartphone of Exp. 1 was also used in this experiment.

\section{Design and Procedure}

The independent variables were Feedback (PS feedback and no-PS), Distance (0mm, $12 \mathrm{~mm}, 24 \mathrm{~mm}, 48 \mathrm{~mm})$ and Size (2.0mm, 2.6mm, $3.2 \mathrm{~mm}$ and $3.8 \mathrm{~mm})$. This design resulted in $2 \times 4 \times 4=32$ Feedback $\times$ Distance $\times$ Size conditions. For each condition, participants perform 52 touch trials with first four touch endpoints ignored as practice. The final design resulted in $48 \times 2 \times 4 \times 4 \times 16=24576$ total trials for analysis.

To counterbalance the order effect of presentation, we first divided participants into two groups, with eight participants performing PS feedback first and the other eight performing no-PS first. We then used two independent Latin squares to counterbalance the order of Distance and Size within each group. The order of presentations was identical in the two groups to ensure fair comparison.

Participants were instructed to perform "as accurately as possible". We explain to participants that the system recorded an error when an endpoint fell outside the visual boundary of the target. Participants had a 15-minutes warmup session to familiarize themselves with the different combinations of Feedback, Distance and Size.

\section{Results}

\section{Target acquisition time and error rate}

There was a significant effect of Size $\left(\mathrm{F}_{3,45}=15.62, \mathrm{P}<.001\right)$ on target selection time. The average selection times were $653 \mathrm{~ms}$ for PS feedback, and $607 \mathrm{~ms}$ for no-PS. The average selection times for increasing distances were $563.2 \mathrm{~ms}$, $576.6 \mathrm{~ms}$, $646.0 \mathrm{~ms}$ and $736.6 \mathrm{~ms}$ respectively. The average selection times for increasing sizes were $679.3 \mathrm{~ms}, 631.5 \mathrm{~ms}$,
$619.5 \mathrm{~ms}$ and $590.1 \mathrm{~ms}$ respectively. Fig. 4 shows the regression results of fitting data to "effective width" model of Fitts' Law [29].

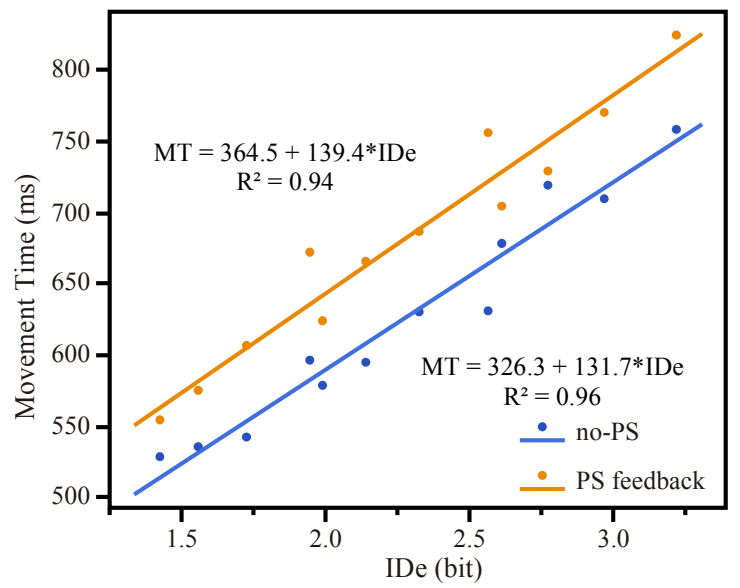

Figure 4. Fit data with the "effective width" model of Fitts' Law

Fig. 5 shows error rates across Feedback $\times$ Distance $\times$ Size conditions. Overall, PS feedback yielded a much lower error rate than no-PS $\left(\mathrm{F}_{1,15}=95.5, \mathrm{P}<.001\right)$. The average error rates were $9.6 \%$ for PS feedback, and $44.2 \%$ for noPS. Post hoc analysis showed PS feedback was significantly more accurate than no-PS for all individual conditions. The effect of Size was also significant $\left(\mathrm{F}_{3,45}=54.58, \mathrm{P}<0.001\right)$. The average error rates for increasing sizes were $41.4 \%$, $30.9 \%, 21.8 \%$ and $13.5 \%$ respectively. That is, increasing size would reduce error rate.

As shown in Fig. 5, as Distance increases, error rate for noPS had a decreasing tendency, while error rate for PS feedback had an increasing tendency.

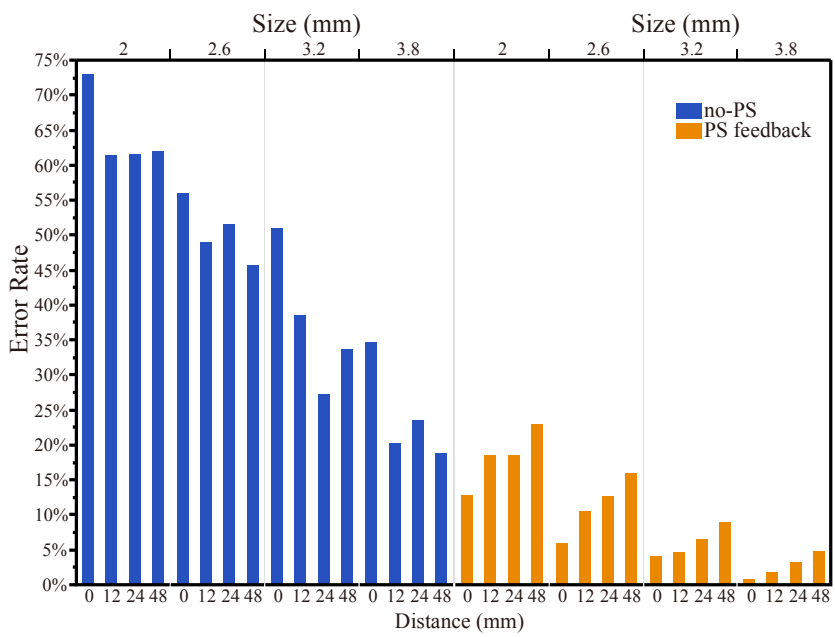

Figure 5. Error rates across different sizes and distances for both PS feedback and no-PS. 


\section{Systematic offset}
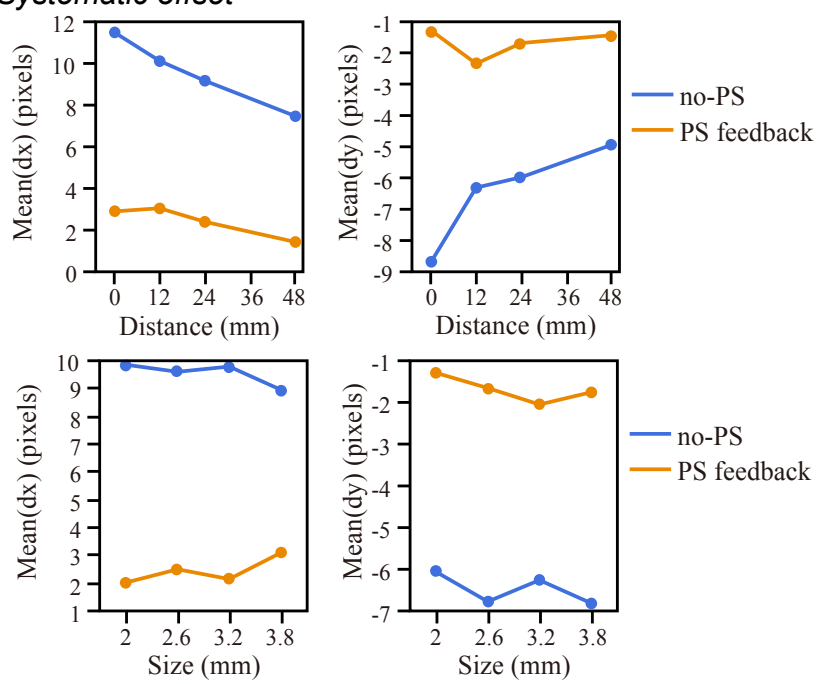

Figure 6. (Top) offset vs. Distance; (Bottom) offset vs. Size

Significant effects of Feedback $\left(\mathrm{x}: \mathrm{F}_{1,15}=28.383, \mathrm{P}<.0001\right.$; $\left.\mathrm{y}: \mathrm{F}_{1,15}=18.597, \mathrm{P}<.001\right)$ were found on both the $\mathrm{x}-$ and $\mathrm{y}$ axis offsets. Systematic offset got smaller as distance increased. No significant effect of Size was found on $\mathrm{x}$ - and $\mathrm{y}$-axis offset $\left(\mathrm{x}: \mathrm{F}_{3,45}=0.033, \mathrm{P}=0.992 ; \mathrm{y}: \mathrm{F}_{3,45}=0.694\right.$, $\mathrm{P}=0.561$ ). Fig. 6 illustrates systematic endpoint offset across Distance and Size.

\section{Endpoint deviation}

There was significant effect of Feedback, Distance and Size on $\mathrm{x}$ - and $\mathrm{y}$-axis deviations (all $\mathrm{P}<.05$ ). Post-selection feedback reduced deviation by $32 \%$ in average. Increasing target size and increasing distance would both increase endpoint deviations (Fig. 7).

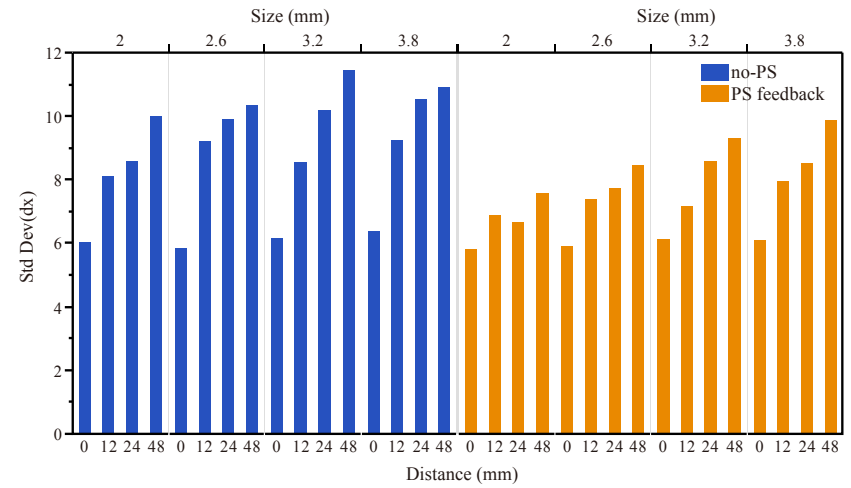

Figure 7. Standard deviations across different sizes and distances for both PS feedback and no-PS.

User feedback

Among all sixteen participants, twelve preferred PS feedback, three preferred no-PS, and one participant had no specific preference. Three who preferred no-PS comments, "The feedback is distracting, especially when the distance is close". One reported, "I tried to adjust as long as I saw the contact point, which made me feel tired".
The majority of participants liked the visual cue, and made comments that "post-selection feedback let me know where I touch exactly", and that "the visualized contact point caught my attention so that I could focus more on the task and perform better". Some stated that post-selection feedback could increase their confidence, and also "make the tedious touching process more interesting".

Participants' subjective feedback suggested distinct adjustment behaviors for smaller targets and larger targets. When target size was small (e.g. $2.0 \mathrm{~mm}$ and $2.6 \mathrm{~mm}$ ), more efforts were paid to adjust finger movement because it was more likely to commit an error. For larger targets (e.g. $3.2 \mathrm{~mm}$ and $3.8 \mathrm{~mm}$ ), participants would intentionally adjust finger movement "when the last tap is really or is about to go beyond the boundary".

At last, fifteen participants self-reported that they made an adjustment based on the prior touch trial at the same location; Fourteen participants preferred post-selection feedback for acquiring smaller-sized targets.

\section{EXPERIMENT THREE: 2D RANDOM TARGETS}

The goal was to examine the effect of post-selection feedback for acquiring 2D targets that were randomly positioned on a smart watch in sitting and standing scenarios, which mimicked a more realistic setting compared to the previous experiments.

\section{Apparatus and Participants}

We recruited 24 participants (16 male and 8 female) aged between 21 and 33 from Tsinghua University. They were all right handed with more than two years' experience on touch screen devices. The experiment system was implemented on a smart watch, Samsung Galaxy Gear SMV700.

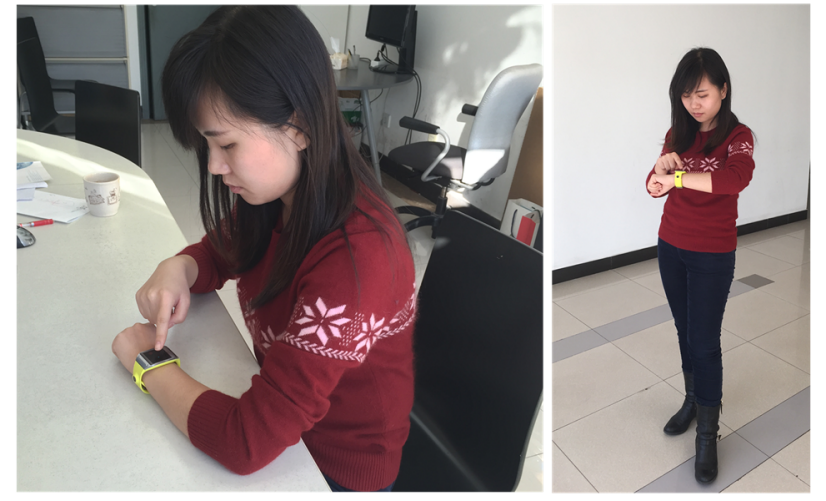

Figure 8. Sitting and standing scenarios for Exp. 3

\section{Design and Procedure}

We used a between subject design for this study. The independent factors were Feedback (PS feedback vs. noPS) Distance $(10 \mathrm{~mm}, 15 \mathrm{~mm}, 20 \mathrm{~mm}, 25 \mathrm{~mm})$, Size $(2.0 \mathrm{~mm}$, $2.6 \mathrm{~mm}, 3.2 \mathrm{~mm}$ and $3.8 \mathrm{~mm}$ ), and Scenario (Sitting and Standing) as shown in Fig. 8. Half of the participants tested PS feedback and the other half tested the no-PS. Each 
participant completed 12 trials for each the condition. In total, we collected $12 \times 4 \times 4 \times 2 \times 24=9216$ trials for analysis.

Two circles were displayed on the watch screen: a reset circle and a target one. The reset circle was always 4 millimeter width. For each trial, the participant first touched the reset circle, and then touched the target circle. Distance was defined as the screen distance between the reset cursor and the target one. Size was defined as the size of the target circle. If the participant missed the target, an audio feedback would be played to notify the error in both PSfeedback and no-PS conditions. The participant repeated the acquisition until succession. After that, a new pair of targets would appear on the screen for the next trial. The system recorded the total time and repetitions of each trial.

\section{Results}

\section{Target acquisition time and error rate}

Feedback $\left(\mathrm{F}_{1,22}=12.85, \mathrm{P}<.005\right)$, Distance $\left(\mathrm{F}_{3,66}=44.36\right.$, $\mathrm{P}<.0001)$ and Size $\left(\mathrm{F}_{3,66}=24.39, \mathrm{P}<.0001\right)$ had significant impact on acquisition time. The mean target acquisition time was $881 \mathrm{~ms}$ for PS feedback and $1470 \mathrm{~ms}$ for no-PS. It was not surprising that PS feedback improved selection accuracy and resulted in less time of completion, because participants needed a smaller number of repetitions to complete each trial. The mean selection times for increasing distances were $886.3 \mathrm{~ms}, 1058.8 \mathrm{~ms}, 1150.9 \mathrm{~ms}$ and $1605.6 \mathrm{~ms}$ respectively. For increasing sizes, the mean selection times were $1982.8 \mathrm{~ms}, 1107.8 \mathrm{~ms}, 889.0 \mathrm{~ms}$ and $722.0 \mathrm{~ms}$ respectively. There was no significant effect of Scenario $\left(\mathrm{F}_{1,22}=0.018, \mathrm{P}=.894\right)$ on selection time. The mean times for sitting and standing were $1180.1 \mathrm{~ms}$ and $1170.7 \mathrm{~ms}$ respectively.

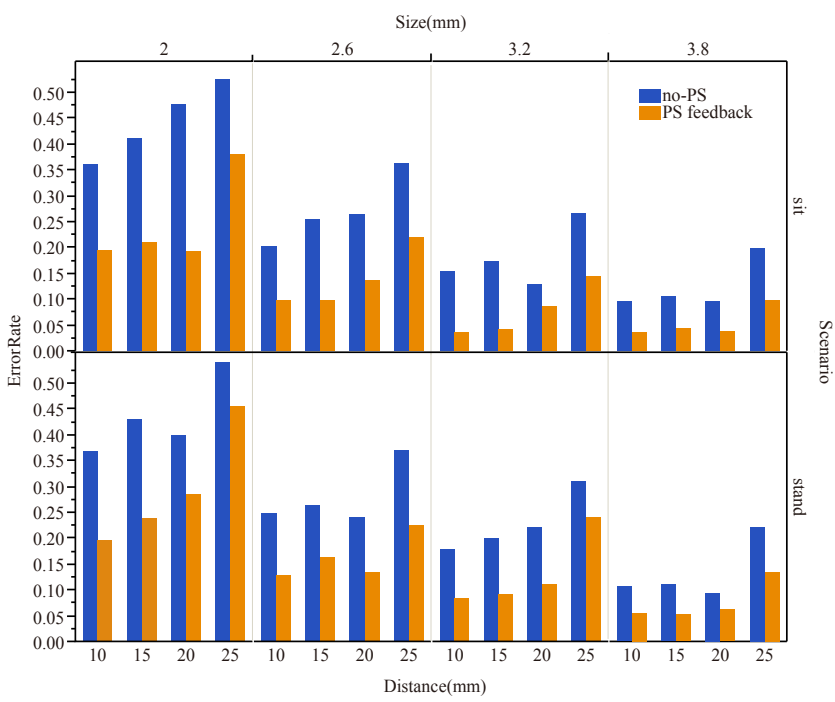

Figure 9. Error rates across different sizes, distances and scenarios for both PS feedback and no-PS.

For each trial, we define the error rate as $1-1 / \mathrm{N}_{\text {Total }}$, where $\mathrm{N}_{\text {Total }}$ was the number of repetitions required to successfully acquire the target. ANOVA showed significant effects of Feedback $\left(\mathrm{F}_{1,22}=223.35, \mathrm{P}<.0001\right)$, Distance $\left(\mathrm{F}_{3,66}=62.10\right.$,
$\mathrm{P}<.0001)$, Size $\left(\mathrm{F}_{3,66}=208.16, \mathrm{P}<.0001\right)$ and Scenario $\left(\mathrm{F}_{1,22}=11.83, \mathrm{P}<.001\right)$ on error rate. The mean error rates of PS feedback and no-PS were $15 \%$ and $26 \%$ respectively. For PS feedback, the mean error rates were $13 \%$ and $17 \%$ for sitting and standing respectively. A possible explanation was the supporting arm (the non-dominant hand wearing the watch) was more stable in the sitting posture than in the standing posture. For no-PS, the mean error rates were $25 \%$ and $27 \%$ for sitting and standing respectively. That is to say, PS feedback improved error rate in both scenarios. From Fig. 9, we can see PS feedback yielded lower error rate than no-PS across all conditions, which was consistent with the result of Exp. 2.

\section{System Offset}

Significant effects of Feedback ( $\mathrm{x}: \mathrm{F}_{1,22}=38.12, \mathrm{P}<.0001$; $\mathrm{y}$ : $\left.\mathrm{F}_{1,22}=11.74, \mathrm{P}<.005\right)$ were found on both the $\mathrm{x}$ - and $\mathrm{y}$-axis offsets. Distance has impact on $\mathrm{x}$-axis $\left(\mathrm{F}_{3,66}=4.72, \mathrm{P}<.005\right)$. No significant effect of Scenario and Size were found on $\mathrm{X}$ and y-axis offset.

\section{Endpoint deviation}

There were significant effects of Feedback, Distance and Scenario on $\mathrm{x}$ - and $\mathrm{y}$-axis deviations (all $\mathrm{P}<.05$ ). No significant effect of Size was found. In average, postselection feedback reduced deviation by $33 \%$.

\section{FEEDBACK ADJUSTMENT MODEL}

Experimental results have already confirmed the benefit of post-selection feedback for acquiring ultra-small targets. The subjective feedbacks from participants also suggested more intentional adjustment to be made with post-selection feedback. However, our understanding about how postselection feedback affects users' finger/point mapping model is still limited. In this section, we take a further step to examine more detailed and quantitative measures about users' touch behavior with and without post-selection feedback, in order to gain a deeper and concrete understanding. To achieve this, we follow a statistical approach. We first examine the correlation between successive touch endpoints, and then quantify intentional adjustment based on the touch data collected from Exp. 1 and 2 .

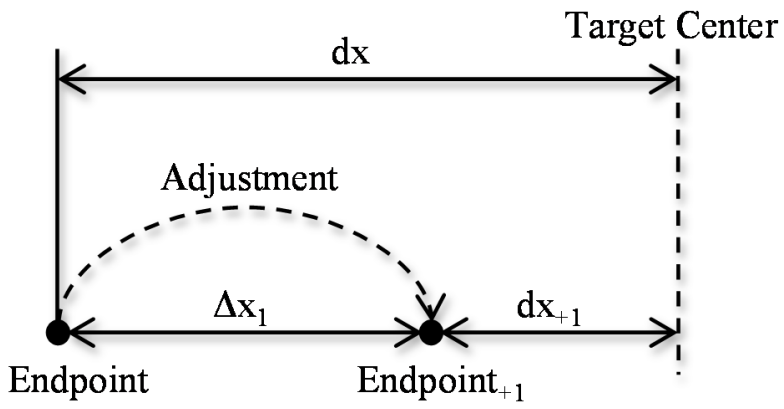

Figure 10. A conceptual representation of users' feedback adjustment behavior 
For clarity, we first define a set of notions to formally describe users' touch behavior. As illustrated in Figure 10, suppose a user is performing a series of target acquisition operations on a touchscreen. We use Endpoint and Endpoint $_{+1}$ to denote two successive touches with respect to the same target. Then, take $\mathrm{x}$ direction for example. We denote the offset of the previous endpoint as $\mathrm{dx}$, and that of the following one as $\mathrm{dx}_{+1}$. We thus quantify the adjustment as $\Delta \mathrm{x}$ as the difference between $\mathrm{dx}_{+1}$ and $\mathrm{dx}$. That is, $\Delta \mathrm{x}=$ $\mathrm{dx}_{+1}-\mathrm{dx}$. The intuition is that if no adjustment is made, the offset of the following touch should be the same as that of the previous one. Note that in Exp. 2, Endpoint and Endpoint $_{+1}$ were intercepted by the other target on the opposite side.

\section{Correlation between touch endpoints}

Hypothesis 1: post-selection feedback affects the correlation between successive touches.

We compute the correlation between $\mathrm{dx}_{+1}$ and $\mathrm{dx}$. Results confirm Hypothesis 1. We observe no correlation for postselection feedback condition, but significant correlation for no feedback condition. Fig. 11 illustrates the endpoint correlation in Exp. 2. Take $\mathrm{x}$-axis for example. Endpoint correlations are 0.61 (Exp. 1) and 0.31 (Exp. 2) for no-PS, and below 0.01 (Exp.1\&2) for PS feedback. This result is against to the common expectation that there should be no correlation if no feedback is present. We discuss this result more in the end of this section. On the other hand, a possible reason for the lower $\mathrm{R}^{2}$ value observed from Exp. 2 is due to the interception of touch on the opposite target. Table 1 summarizes more detailed correlation results for each condition in Exp. 2.

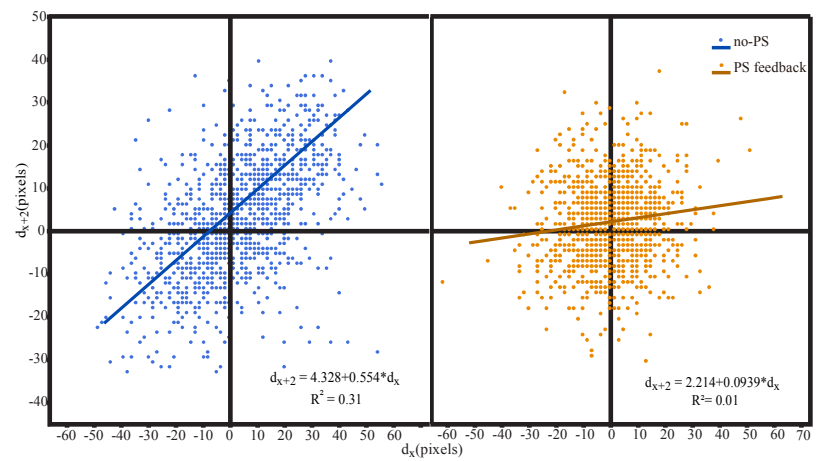

Figure 11: Endpoint correlation in Exp. 2.

Adjustment behavior analysis

Hypothesis 2: Users make more intentional adjustment with post-selection feedback.

In Figure 11, if $\Delta \mathrm{x}_{1}$ and $\mathrm{dx}$ are of opposite signs, it means the adjustment is made to compensate the offset of the previous touch trial. We note it as a positive adjustment. However, if $\mathrm{dx}$ and $\Delta \mathrm{x}_{1}$ are of the same sign, it means Endpoint $_{+1}$ is even more distant from target center than Endpoint. We note it a negative adjustment.
For actual task, $\Delta \mathrm{x}_{1}$ is a random variable. It is the combined result of participants' intentional/planned adjustment and uncontrolled movement noise $(\mathrm{Mean}=0)$ that links to the randomness of human's cognitive and motor system. Therefore, we can quantify participants' adjustment behavior based on the statistics of $\Delta \mathrm{x}_{1}$. Specifically, mean of $\Delta \mathrm{x}_{1}$ is the intentional adjustment that has been made; the standard deviation of $\Delta \mathrm{x}_{1}$ is a measure of the amplitude of uncontrolled noise when making the adjustment.

Fig. 12 illustrates $\Delta \mathrm{x}_{1} \sim \mathrm{dx}$ and $\Delta \mathrm{y}_{1} \sim$ dy for both PS feedback and no-PS of Exp. 1. We define positive adjustment ratio $(P A R)$ as the ratio of data points fallen into the second and forth quadrants, whose $\Delta \mathrm{x}_{1}\left(\Delta \mathrm{y}_{1}\right)$ and $\mathrm{dx}(\mathrm{dy})$ are of opposite signs. For PS feedback, positive adjustment ratios are $77 \%$ and $71 \%$ on the $\mathrm{x}$ and $\mathrm{y}$ axes respectively. For noPS, positive adjustment ratios are $56 \%$ and $55 \%$ on the $x$ and $y$ axes respectively. This means with PS feedback, participants make 33\% more positive adjustments than noPS.
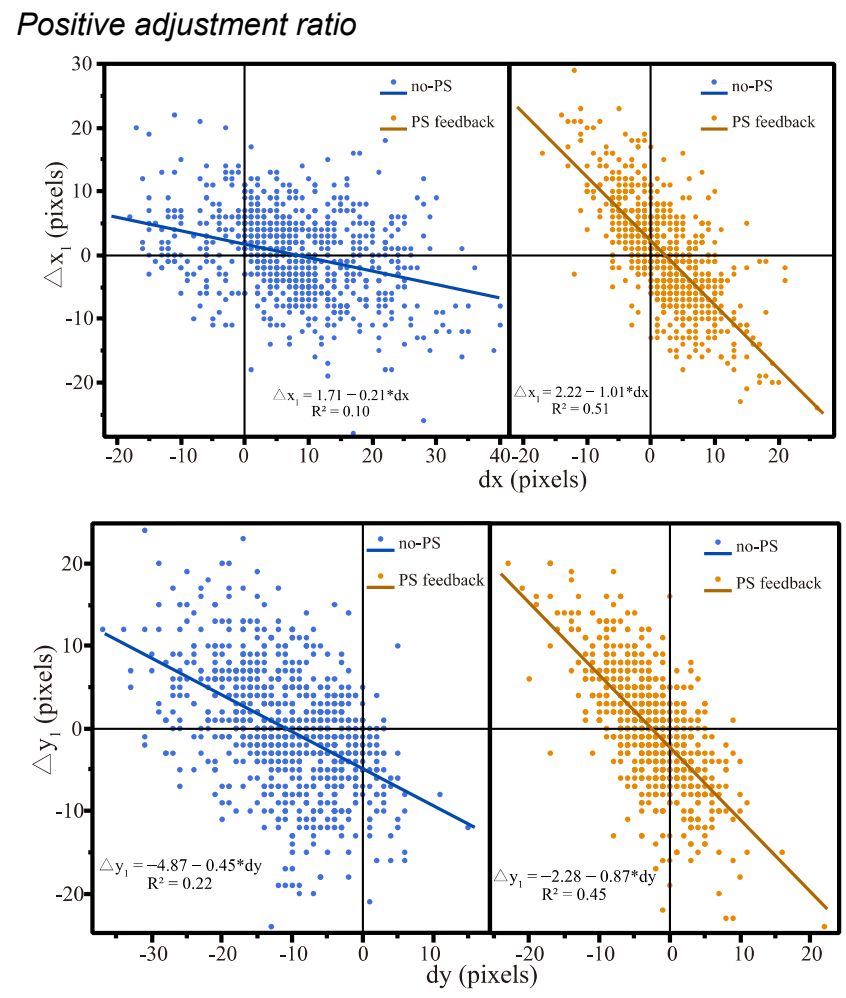

Figure 12. $\Delta \mathbf{x}_{1} \sim \mathrm{dx}$ and $\Delta \mathrm{y}_{1} \sim \mathrm{dy}$ for PS feedback and no-PS.

Fig. 13 shows positive adjustment ratio across all $\mathrm{dx}$ values for both PS feedback and no-PS. We look at PS feedback first (the orange dots and curve). As shown, more positive adjustments can be made as $|\mathrm{dx}|$ increased. The positive adjustment ratio achieves $100 \%$ when $\mathrm{dx}$ is below -7.3 pixels or above 12 pixels. The reason is obvious since the greater the amplitude of the offset of the previous trial, the easier for participants to notice the offset and make a positive adjustment in the subsequent trial. 


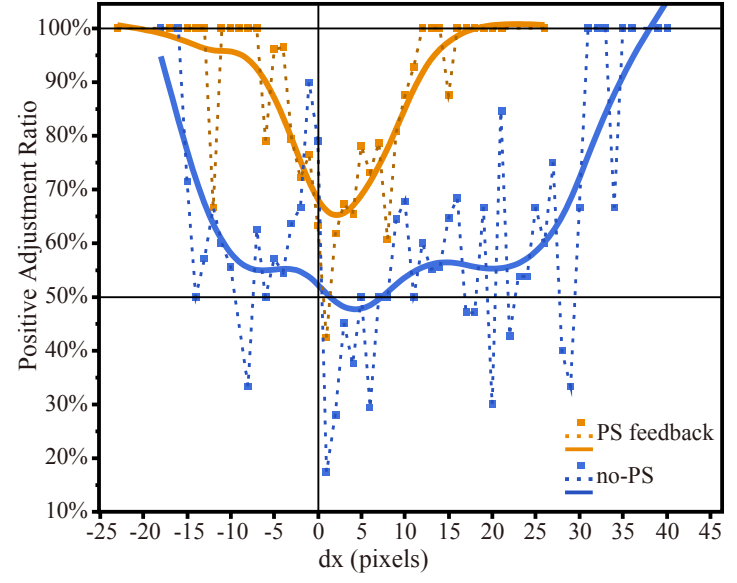

Figure 13. Positive adjustment ratio across dx for PS feedback and no-PS conditions.

Interestingly, we observe the same trend for no-PS. Participants can also make $100 \%$ positive adjustments when the amplitude of offset was large. This suggests that intentional adjustment indeed happens even if no postselection feedback is present. According to our observation, we suspect in no-PS condition, participants can still estimate, although not accurately, the touch offset according to their finger/point mapping in the mental model, especially when the amplitude of the offset was large.

Finally, PS feedback consistently yields higher positive adjustment ratio across $\mathrm{dx}$, and achieves $100 \%$ much faster on both sides. This provides our first quantitative evidence that participants can make more intentional adjustment with post-selection feedback.

\section{Linear regression}

We also apply linear fit to the data points. Regression results are shown in Fig. 15. Next, we interpret the meanings of fitting parameters (slope, $\mathrm{x}$-intercept and $\mathrm{R}^{2}$ value) in the context of participants' adjustment behavior.

$\mathrm{X}$-intercept of the regression line denotes the average $\mathrm{dx}$ where the positive adjustment and negative adjustment are equal. For PS feedback, $x-$ and y-intercepts are 2.2 pixels and -2.6 pixels respectively. For no-PS, $x-$ and y-intercepts are 8.1 pixels and -10.8 pixels respectively. Comparing these numbers to systematic offsets of endpoints (see results of Exp. 1), we see a high consistency. This suggests that participants make adjustment toward the perceived target center (the systematic offset), rather than the nominal target center, in regardless of feedbacks.

The slope of the regression lines characterizes the degree to which adjustment is made with respect to the amplitude of the offset. A slope of -1 represents ideal adjustment behavior whose average adjustment exactly compensates the offset of the very last touch trial. On the other end, a slope of 0 means the adjustment is totally random; in other words, the participant has no idea about where the target center is. In Exp. 1, for PS feedback, the slopes are -1.01 for $\mathrm{x}$ axis, and -0.87 for $\mathrm{y}$ axis; for no-PS, the slopes are much flatter, -0.21 for $\mathrm{x}$ axis, and -0.45 for $\mathrm{y}$ axis. These results show that with post-selection feedback, participants are quite aware of the target center, and thus can perform effective adjustment. For no-PS condition, the target center seems to constantly migrate during the task.

The goodness of model fit ( $\mathrm{R}^{2}$ value) is analogous to signalto-noise ratio of participants performing the adjustment movement. Again, $\mathrm{R}^{2}$ value of PS-feedback $(0.51$ for $\mathrm{x}$ direction; 0.45 for $\mathrm{y}$ direction) is much higher than that of no-PS condition ( 0.1 for $\mathrm{x}$ direction; 0.22 for $\mathrm{y}$ direction). This result suggests that with post-selection feedback, participants make more precise adjustment than with no feedback.

Table 2 summarizes the results for Exp.2, which reports the four measures discussed above. Due to limitation of length, we only report results on X-axis. Results showed that in all conditions, the four investigated measures of PS feedback are superior to those of no-PS in terms of manifesting intentional adjustment behavior. This indicates participants make more intentional adjustments with Point in regardless of movement distance and target size.

\begin{tabular}{|l|l|l|l|l|l|l|l|l|l|}
\hline \multicolumn{4}{|l|}{} & \multicolumn{3}{|l}{ No Feedback } & \multicolumn{3}{|l|}{ Post-selection Feedback } \\
\hline S & D & slope & $\begin{array}{l}\text { interce } \\
\text { pt-x }\end{array}$ & $\mathrm{R}^{2}$ & PAR & slope & $\begin{array}{l}\text { interce } \\
\text { t-x }\end{array}$ & $\mathrm{R}^{2}$ & PAR \\
\hline 2 & 0 & -0.32 & 11.68 & 0.15 & 0.55 & -0.94 & 2.62 & 0.47 & 0.74 \\
\hline 2 & 12 & -0.37 & 9.81 & 0.17 & 0.58 & -0.94 & 2.15 & 0.47 & 0.74 \\
\hline 2 & 24 & -0.44 & 10.16 & 0.21 & 0.57 & -0.93 & 1.91 & 0.47 & 0.73 \\
\hline 2 & 48 & -0.61 & 8.28 & 0.29 & 0.63 & -0.92 & 1.38 & 0.47 & 0.77 \\
\hline 2.6 & 0 & -0.28 & 10.89 & 0.14 & 0.57 & -0.85 & 2.72 & 0.42 & 0.69 \\
\hline 2.6 & 12 & -0.36 & 10.63 & 0.18 & 0.60 & -0.82 & 3.47 & 0.40 & 0.70 \\
\hline 2.6 & 24 & -0.43 & 10.02 & 0.22 & 0.63 & -0.92 & 2.85 & 0.42 & 0.71 \\
\hline 2.6 & 48 & -0.55 & 7.52 & 0.28 & 0.65 & -0.97 & 1.01 & 0.47 & 0.75 \\
\hline 3.2 & 0 & -0.26 & 13.19 & 0.14 & 0.53 & -0.87 & 2.81 & 0.40 & 0.71 \\
\hline 3.2 & 12 & -0.38 & 11.70 & 0.19 & 0.57 & -0.75 & 2.80 & 0.37 & 0.73 \\
\hline 3.2 & 24 & -0.59 & 8.16 & 0.29 & 0.63 & -0.80 & 1.90 & 0.40 & 0.72 \\
\hline 3.2 & 48 & -0.54 & 6.96 & 0.26 & 0.64 & -0.86 & 1.15 & 0.42 & 0.74 \\
\hline 3.8 & 0 & -0.34 & 11.36 & 0.17 & 0.53 & -0.83 & 3.57 & 0.38 & 0.68 \\
\hline 3.8 & 12 & -0.37 & 9.24 & 0.19 & 0.60 & -0.74 & 3.79 & 0.36 & 0.69 \\
\hline 3.8 & 24 & -0.52 & 8.77 & 0.26 & 0.61 & -0.92 & 2.97 & 0.45 & 0.71 \\
\hline 3.8 & 48 & -0.60 & 7.37 & 0.30 & 0.67 & -0.90 & 2.14 & 0.43 & 0.75 \\
\hline
\end{tabular}

Table 2. Adjustment behavior analysis for Exp. 2. PAR is shorted for positive adjustment ratio.

\section{Summary}

The analysis of endpoint correlation and adjustment behavior let us have an insight into users' touch behavior. With post-selection feedback, users are aware of actual 
touch contact point each time after they lift their finger. This makes users' finger/point mapping model explicit and more accurate. Users can also make more effective intentional adjustment to touch more accurately. In contrast, with no feedback, users have to rely on their implicit mental model of finger/point mapping to perform touch. Unfortunately, according to the results, we can see the mapping is not either accurate or stable. The perceived contact point constantly migrates due to the complexity of touching model. This accounts for the significant endpoint correlation as well as greater endpoint deviation for nofeedback condition.

To our knowledge, this is the first investigation on endpoint correlation in order to provide an insight into users' touch behavior. The results can not only complement our understanding about users' touch behavior, but also potentially help researchers to develop more sophisticated movement control models of touch, and to develop target prediction algorithms by considering endpoint correlation, e.g. augmenting the spatial model of touch input in software keyboard algorithm.

\section{CONCLUSION AND FUTURE WORK}

In this paper, we investigate the effects of post-selection feedback for acquiring ultra-small targets sized from $2 \mathrm{~mm}$ $4 \mathrm{~mm}$. We research the effects of movement distance and target size on performance of post-selection feedback in terms of target selection time, accuracy, endpoint offset and deviation. Three experiments with increasing complexity are designed to accommodate the relevant factors, which allow us to examine the performance of post-selection feedback systematically.

The overall results show that for all target sizes and movement distances, post-selection feedback can reduce target acquisition errors by $74.2 \%$ with a compromise of target selection time no more than $10 \%$. We also examine the effects of movement distance and target size on systematic offset and deviation of touch endpoints. Specifically, increasing movement distance will significantly but slightly reduce systematic offset, and significantly increases endpoint deviation. Increasing target size will increase endpoint deviation but will not affect systematic offset.

Moreover, we examine correlation between successive touches to research users' feedback adjustment behavior. Through proper quantification, we successfully demonstrate the presence of feedback adjustment behavior using objective measures, consolidating users' subjective feedback. Our method contrasts to previous research, which usually treated target acquisition trials in a serial task independently. We thus argue for the necessity of examining correlation between successive trials for future work to gain deeper understandings about users' target acquisition behavior.
Based on our results, we believe the benefit of postselection feedback comes from two aspects: an improved understanding about finger/point mapping and dynamic adjustment of finger movement to compensate offset.

Finally, there are a number of future directions of this work, including 1) examining the impact of feedback visualization on touch performance [18], 2) investigating the use of postselection feedback for more realistic tasks on ultra-small devices (e.g. text entry on QWERTY keyboard), 3) comparing the performance of post-selection feedback in static and mobile environment, 4) digging into the correlation between successive touch trials to gain more understanding about human's touch behavior, and 5) researching the long-term effect of post-selection feedback for improving users' touch ability.

\section{ACKNOWLEDGMENTS}

This work is supported by the Natural Science Foundation of China under Grant 61303076 and 61272230, Tsinghua University Research Funding 20151080408 and 20151300771

\section{REFERENCES}

1. Pär-Anders Albinsson and Shumin Zhai. 2003. High precision touch screen interaction. In Proceedings of the SIGCHI Conference on Human Factors in Computing Systems (CHI '03), 105-112. http://dx.doi.org/10.1145/642611.642631

2. Oscar Kin-Chung Au, Xiaojun $\mathrm{Su}$, and Rynson W.H. Lau. 2014. LinearDragger: a Linear Selector for Onefinger Target Acquisition. In Proceedings of the SIGCHI Conference on Human Factors in Computing Systems (CHI '14), 2607-2616. http://dx.doi.org/10.1145/2556288.2557096

3. Shiri Azenkot and Shumin Zhai. 2012. Touch behavior with different postures on soft smartphone keyboards. In Proceedings of the 14th international conference on Human-computer interaction with mobile devices and services (MobileHCI '12), 251-260. http://dx.doi.org/10.1145/2371574.2371612

4. Patrick Baudisch and Gerry Chu. 2009. Back-of-device interaction allows creating very small touch devices. In Proceedings of the SIGCHI Conference on Human Factors in Computing Systems (CHI '09), 1923-1932. http://dx.doi.org/10.1145/1518701.1518995

5. Xiaojun Bi, Yang Li, and Shumin Zhai. 2013. FFitts law: modeling finger touch with fitts' law. In Proceedings of the SIGCHI Conference on Human Factors in Computing Systems (CHI '13), 1363-1372. http://dx.doi.org/10.1145/2470654.2466180 
6. Stephen Brewster, Faraz Chohan, and Lorna Brown. 2007. Tactile feedback for mobile interactions. In Proceedings of the SIGCHI Conference on Human Factors in Computing Systems (CHI '07), 159-162. http://dx.doi.org/10.1145/1240624.1240649

7. Alex Butler, Shahram Izadi, and Steve Hodges. 2008. SideSight: multi-"touch" interaction around small devices. In Proceedings of the 21st annual ACM symposium on User interface software and technology (UIST '08), 201-204. http://dx.doi.org/10.1145/1449715.1449746

8. Andy Cockburn, David Ahlström, and Carl Gutwin. 2012. Understanding performance in touch selections: Tap, drag and radial pointing drag with finger, stylus and mouse. Int. J. Hum.-Comput. Stud. 70, 3 (March 2012), 218-233. http://dx.doi.org/10.1016/j.ijhcs.2011.11.002

9. Chris Harrison and Scott E. Hudson. 2009. Abracadabra: wireless, high-precision, and unpowered finger input for very small mobile devices. In Proceedings of the 22nd annual ACM symposium on User interface software and technology (UIST '09), 121124. http://dx.doi.org/10.1145/1622176.1622199

10. Niels Henze, Enrico Rukzio, and Susanne Boll. 2012. Observational and experimental investigation of typing behaviour using virtual keyboards for mobile devices. In Proceedings of the SIGCHI Conference on Human Factors in Computing Systems (CHI '12), 2659-2668. http://dx.doi.org/10.1145/2207676.2208658

11. Eve Hoggan and Stephen Brewster. 2007. Designing audio and tactile crossmodal icons for mobile devices. In Proceedings of the 9th international conference on Multimodal interfaces (ICMI '07), 162-169. http://dx.doi.org/10.1145/1322192.1322222

12. Eve Hoggan, Stephen A. Brewster, and Jody Johnston. 2008. Investigating the effectiveness of tactile feedback for mobile touchscreens. In Proceedings of the SIGCHI Conference on Human Factors in Computing Systems (CHI '08), 1573-1582. http://dx.doi.org/10.1145/1357054.1357300

13. Christian Holz and Patrick Baudisch. 2010. The generalized perceived input point model and how to double touch accuracy by extracting fingerprints. In Proceedings of the SIGCHI Conference on Human Factors in Computing Systems (CHI '10), 581-590. http://dx.doi.org/10.1145/1753326.1753413

14. Christian Holz and Patrick Baudisch. 2011. Understanding touch. In Proceedings of the SIGCHI Conference on Human Factors in Computing Systems (CHI '11), 2501-2510. http://dx.doi.org/10.1145/1978942.1979308
15. Luis A. Leiva, Alireza Sahami, Alejandro Catala, Niels Henze, and Albrecht Schmidt. 2015. Text Entry on Tiny QWERTY Soft Keyboards. In Proceedings of the 33rd Annual ACM Conference on Human Factors in Computing Systems (CHI '15), 669-678. http://dx.doi.org/10.1145/2702123.2702388

16. Stephen Oney, Chris Harrison, Amy Ogan, and Jason Wiese. 2013. ZoomBoard: a diminutive qwerty soft keyboard using iterative zooming for ultra-small devices. In Proceedings of the SIGCHI Conference on Human Factors in Computing Systems (CHI '13), 27992802. http://dx.doi.org/10.1145/2470654.2481387

17. Anna Ostberg and Nada Matic. 2015. Hover Cursor: Improving Touchscreen Acquisition Of Small Targets With Hover-enabled Pre-selection. In Proceedings of the 33rd Annual ACM Conference Extended Abstracts on Human Factors in Computing Systems (CHI EA '15), 1723-1728. http://dx.doi.org/10.1145/2702613.2732903

18. Jeeyea Park and Kwang-Hee Han. 2011. Effect of Target Size and Duration of Visual Feedback on Touch Screen. HCI International 2011-Posters' Extended Abstracts, 252-256.

19. Richard L. Potter, Linda J. Weldon, and Ben Shneiderman. 1988. Improving the accuracy of touch screens: an experimental evaluation of three strategies. In Proceedings of the SIGCHI Conference on Human Factors in Computing Systems (CHI '88), 27-32. http://dx.doi.org/10.1145/57167.57171

20. Ivan Poupyrev and Shigeaki Maruyama. 2003. Tactile interfaces for small touch screens. In Proceedings of the 16th annual ACM symposium on User interface software and technology (UIST '03), 217-220. http://dx.doi.org/10.1145/964696.964721

21. Anne Roudaut, Stéphane Huot, and Eric Lecolinet. 2008. TapTap and MagStick: improving one-handed target acquisition on small touch-screens. In Proceedings of the working conference on Advanced visual interfaces (AVI '08), 146-153. http://dx.doi.org/10.1145/1385569.1385594

22. Andrew Sears. 1991. Improving touchscreen keyboards: Design issues and a comparison with other devices. Interact. Comput. 3, 3: 253-269.

23. Chao-Huai Su, Liwei Chan, Chien-Ting Weng, RongHao Liang, Kai-Yin Cheng, and Bing-Yu Chen. 2013. NailDisplay: bringing an always available visual display to fingertips. In Proceedings of the SIGCHI Conference on Human Factors in Computing Systems (CHI '13), 1461-1464.

http://dx.doi.org/10.1145/2470654.2466193 
24. Ahsanullah, Suziah Sulaiman, Ahmad Kamil Mahmood, and Muzafar Khan. 2015. Investigation of fingertip contact area and shape for precise target selection on multi-touch screen. In Proceedings of the 9th International Conference on Ubiquitous Information Management and Communication (IMCOM '15), Article 31, 6 pages.

http://dx.doi.org/10.1145/2701126.2701209

25. Keith Vertanen, Haythem Memmi, Justin Emge, Shyam Reyal, and Per Ola Kristensson. 2015. VelociTap: Investigating Fast Mobile Text Entry using SentenceBased Decoding of Touchscreen Keyboard Input. In Proceedings of the 33rd Annual ACM Conference on Human Factors in Computing Systems (CHI '15), 659668.

http://dx.doi.org/10.1145/2702123.2702135

26. Daniel Vogel and Patrick Baudisch. 2007. Shift: a technique for operating pen-based interfaces using touch. In Proceedings of the SIGCHI Conference on Human Factors in Computing Systems (CHI '07), $657-$ 666.

http://dx.doi.org/10.1145/1240624.1240727
27.Feng Wang and Xiangshi Ren. 2009. Empirical evaluation for finger input properties in multi-touch interaction. In Proceedings of the SIGCHI Conference on Human Factors in Computing Systems (CHI '09), 1063-1072.

http://dx.doi.org/10.1145/1518701.1518864

28. Daniel Wigdor, Sarah Williams, Michael Cronin, Robert Levy, Katie White, Maxim Mazeev, and Hrvoje Benko. 2009. Ripples: utilizing per-contact visualizations to improve user interaction with touch displays. In Proceedings of the 22nd annual ACM symposium on User interface software and technology (UIST '09), 312.

http://dx.doi.org/10.1145/1622176.1622180

29. Shumin Zhai, Jing Kong, and Xiangshi Ren. 2004. Speed-accuracy tradeoff in Fitts' law tasks: on the equivalency of actual and nominal pointing precision. Int. J. Hum.-Comput. Stud. 61, 6 (December 2004), 823856.

http://dx.doi.org/10.1016/j.ijhcs.2004.09.007 